\title{
ON THE CONNECTEDNESS OF THE REAL PART OF MODULI SPACES OF VECTOR BUNDLES ON REAL ALGEBRAIC SURFACES
}

\author{
EDOARDO BALLICO
}

(Received 19 August 1998; revised 5 May 1999)

Communicated by W. Newmann

\begin{abstract}
Let $X$ be a smooth projective surface with $q(X)=0$ defined over $\mathbb{R}$ and $M\left(X ; r ; c_{1}, c_{2} ; H\right)$ the moduli space of $H$-stable rank $r$ vector bundles on $X$ with Chern classes $c_{1}$ and $c_{2}$. Assume either $r=3$ and $X(\mathbb{R})$ connected or $r=3$ and $X(\mathbb{R})=\emptyset$ or $r=2$ and $X(\mathbb{R})=\emptyset$. We prove that quite often $M$ is connected.
\end{abstract}

1991 Mathematics subject classification (Amer. Math. Soc.): primary 14P99, $14 \mathrm{~J} 60$.

\section{Introduction}

In this paper we continue a topic considered in [B2]: the description of the real part of a moduli scheme of vector bundles on a smooth complete algebraic surface defined over $\operatorname{Spec}(\mathbb{R})$.

Let $X$ be a smooth complete connected algebraic surface defined over $\operatorname{Spec}(\mathbb{R})$. Let $\sigma$ be the anti-holomorphic involution of $X$ inducing its real structure. Fix integers $r$ and $c_{2}$ with $r \geq 2$ and two real line bundles $H$ and $c_{1}$ on $X$ with $H$ ample. Let $M\left(X ; r ; c_{1}, c_{2} ; H\right)$ be the scheme (defined over $\left.\operatorname{Spec}(\mathbb{R})\right)$ of all $H$-stable rank $r$ vector bundles on $X$ with Chern classes $c_{1}$ and $c_{2}$. The Galois action induces an anti-holomorphic involution $\sigma$ on $M\left(X ; r ; c_{1}, c_{2} ; H\right)(\mathbb{C})$. Let $M\left(X ; r ; c_{1}, c_{2} ; H\right)(\sigma)$ be its fixed locus (with the induced Euclidean topology, unless otherwise stated), that is the real part of the scheme $M\left(X ; r ; c_{1}, c_{2} ; H\right) . M\left(X ; r ; c_{1}, c_{2} ; H\right)(\sigma)$ parametrizes the bundles which are isomorphic to their complex conjugate.

(C) 2000 Australian Mathematical Society 0263-6115/2000\$A2.00+0.00 
In this paper we study the connectedness of $M\left(X ; r ; c_{1}, c_{2} ; H\right)(\sigma)$. A more interesting subset of $M\left(X ; r ; c_{1}, c_{2} ; H\right)$ (at least from the point of view of moduli functors) is the subset $M\left(X ; r ; c_{1}, c_{2} ; H\right)$ (real) parametrizing the real vector bundles, that is the vector bundles defined over $\operatorname{Spec}(\mathbb{R})$. Notice that $M\left(X ; r ; c_{1}, c_{2} ; H\right)(\mathrm{real}) \subseteq$ $M\left(X ; r ; c_{1}, c_{2} ; H\right)(\sigma)$. We believe that quite often $M\left(X ; r ; c_{1}, c_{2} ; H\right)($ real $) \neq M(X$; $\left.r ; c_{1}, c_{2} ; H\right)(\sigma)$, but we do not have any example with $X(\mathbb{R}) \neq \emptyset$ concerning this moduli problem. As explained in step (b) of the proof of Theorem 1.6, Theorem 1.7 and Theorem 1.8, it is technically much easier to work with $M\left(X ; r ; c_{1}, c_{2} ; H\right)(\sigma)$ than with $M\left(X ; r ; c_{1}, c_{2} ; H\right)(\mathrm{real})$. We believe that in many cases in which $M\left(X ; r ; c_{1}, c_{2}\right.$; $H)(\sigma)$ is connected $M\left(X ; r ; c_{1}, c_{2} ; H\right)(\mathrm{real})$ is not connected. We consider $M(X ; r$; $\left.c_{1}, c_{2} ; H\right)(\sigma)$ as a very natural partial compactification of $M\left(X ; r ; c_{1}, c_{2} ; H\right)$ (real). Very often it is quite easy to prove the existence of natural partial compactifications of $M\left(X ; r ; c_{1}, c_{2} ; H\right)($ real) which are connected (see [B2, Proposition 5.3]). For a philosophical justification of this occurrence, see the discussion after the statement of Theorem 1.8. We stress that we consider only surfaces with $q(X)=0$. We believe that for 'most' surfaces with $q(X)>0$ for fixed $r, H$ and $c_{1}$ for all large $c_{2}$ $M\left(X ; r ; c_{1}, c_{2} ; H\right)(\mathrm{real})$ and $M\left(X ; r ; c_{1}, c_{2} ; H\right)(\sigma)$ are disconnected, but we do not know how to prove results in this direction. For the computation of one example see Section 2.

In this paper we give an abstract set-up for this problem. We will list four conditions (see Conditions (A), (B), (C), (D) listed in Section 1) on the data $X, r, c_{i}, H$. When these conditions are satisfied by a $\sigma$-invariant connected component $Y$ of $M\left(X ; r ; c_{1}, c_{2} ; H\right)(\mathbb{C})$ we will prove (see Theorem 1.6 and Theorem 1.7$)$ that the fixed locus $Y(\sigma)$ is connected and not empty if either $X(\mathbb{R})$ is empty or $X(\mathbb{R})$ is connected, $r \geq 3$ and a parity condition is satisfied. When $X(\mathbb{R})$ has $s \geq 2$ connected components, $r \geq 3$ and these conditions are satisfied, we will show (see Theorem 1.8) that $Y(\sigma)$ has at most $3\left(2^{s-1}\right)$ connected components. Furthermore, these conditions imply the existence of a $\sigma$-invariant component. If $r=2$ or $r=3$ we translate part of these conditions into numerical conditions (see Conditions (AB1), (AB2), (C0), (D0), (D1) and (D2)) which may be checked in special cases. In [B2, Theorem 5.1], the technically very easy case $X=P^{2}$ and $r=3$ was done.

In Section 2 we give a key example of surface $X$ with very good $M\left(X ; 2 ; c_{1}, c_{2} ; H\right)$ (that is everywhere smooth and satisfying Conditions (A), (B), (C) and (D)) with $M\left(X ; 2 ; c_{1}, c_{2} ; H\right)(\sigma)$ not connected. In this example $X=C \times P^{1}$ with $C$ an elliptic curve with two circles as real part $C(\mathbb{R})$. In Section 3 we show how to check the axioms in the case of Segre-Hirzebruch surfaces $F_{e}, e \geq 0$. Essentially, we believe that the axioms (A), (B), (C) and (D) are true if $X$ is a rational surface, $r=2$ or $r=3$ and (for fixed $c_{1}$ and $H$ ) $c_{2}$ is very large. However, as shown by the examples given in Section 3 the axioms (at least for $r=2$ and $r=3$ ) may be translated only in very messy numerical conditions. We believe that this is not due to our approach, but to 
the nature of the problem considered here.

\section{The main results}

First, we list several notations and conventions. We fix $X, r, c_{1}, c_{2}, H, \sigma$ as in the introduction; if $F$ is a sheaf on $X$, set $H^{i}(F):=H^{i}(X, F)$ and $h^{i}(F):=h^{i}(X, F)$; set $K:=K_{X}, O:=O_{X}, \chi:=\chi(O), q:=q(X):=h^{1}(O), p_{g}:=p_{g}(X):=h^{0}(K)=$ $h^{2}(O)$. If $A$ is a closed subscheme of a scheme $B, I_{A, B}$ will denote the ideal sheaf of $A$ in $B$; set $I_{A}:=I_{A, X}$. We do not distinguish between a Cartier divisor on $X$ and the associated line bundle; for both objects we will use freely both the additive and the multiplicative notation. When the action of $\sigma$ on $X$ induces an anti-holomorphic involution on another scheme $Y, Y(\sigma)$ will denote its fixed locus. We use (at least for the numerical computations) the convention that 'stability' means 'stability in the sense of Mumford and Takemoto'. We use often in several different situations the following objects:

$$
\begin{gathered}
0 \rightarrow(r-1) O E \rightarrow I_{Z}(L) \rightarrow 0 \\
L:=M \otimes r \otimes c_{1} \quad \text { and } \quad D_{2}:=c_{2}+(r-1) M c_{1}+(r(r-1) / 2) M^{2} \in Z
\end{gathered}
$$

with $L$ and $M$ line bundles and $Z$ a 0 -dimensional subscheme of $X$. Notice that for every rank $r$ bundle $B$ with $c_{i}(B)=c_{t}$ we have $c_{1}(B \otimes M) \cong L$ and $c_{2}(B \otimes M)=d_{2}$. If $L$ and $Z$ are defined over $\operatorname{Spec}(\mathbb{R})$, then the set of extensions (1) over $\operatorname{Spec}(\mathbb{C})$ is the complex vector space associated to a real vector space. The exact sequence (1) will be an exact sequence both if we view the schemes and sheaves over $\operatorname{Spec}(\mathbb{R})$ or over $\operatorname{Spec}(\mathbb{C})$. If $L$ and $Z$ are defined over $\operatorname{Spec}(\mathbb{R})$ the open subset $U$ (respectively $U^{\prime}$ ) of the complex vector space of all the extensions (1) with $E$ locally free (respectively locally free and stable) is defined over $\operatorname{Spec}(\mathbb{R})$. We stress that a bundle defined over $\operatorname{Spec}(\mathbb{R})$ is semistable over $\operatorname{Spec}(\mathbb{R})$ if and only if it is semistable over $\operatorname{Spec}(\mathbb{C})$ ([L, Proposition 3, p. 97]). We will always consider the condition of stability over $\operatorname{Spec}(\mathbb{C})$. For real bundles this is a stronger condition than stability over $\operatorname{Spec}(\mathbb{R})$. However, it is easy to check as in [L, Proposition 3, p. 97, proof of case 1], that the only rank 2 real bundles which are stable over $\operatorname{Spec}(\mathbb{R})$ but not stable over $\operatorname{Spec}(\mathbb{C})$ are the bundles $A \oplus \sigma^{*}(A)$ with $A \in(\operatorname{Pic}(X)(\mathbb{C}) \backslash \operatorname{Pic}(X)(\mathbb{R}))$. If $L$ and $Z$ are only assumed to be $\sigma$-invariant, then the complex vector space of all the extensions has a real structure and the open subset $U$ (respectively $U^{\prime}$ ) parametrizing the locally free (respectively the locally free and stable) sheaves $E$ is $\sigma$-invariant.

1.1 We fix an integer $r \geq 2$ (the rank), a real line bundle $c_{1} \in \operatorname{Pic}(X)(\mathbb{R})$, an integer $c_{2}$ and a Zariski open subset $Y$ of $M\left(X ; r ; c_{1}, c_{2} ; H\right)(\mathbb{C}), Y \neq \emptyset$ with $Y_{\text {red }}$ irreducible. Unless otherwise stated, $Y$ will be defined over $\operatorname{Spec}(\mathbb{R})$. We consider the following 
conditions (A), (B), (C) and (D) which these data may have. These conditions will be assumed to be true in our connectedness Theorem 1.6, Theorem 1.7 and Theorem 1.8.

Condition (A): $Y$ is smooth.

Condition (B) (for the line bundle $M$ ): Fix a real line bundle $M$ on $X$ and take $L, d_{2}$ given by (2). Notice that for any $B \in M\left(X ; r ; c_{1}, c_{2} ; H\right)$, we have $B \otimes M \in$ $M\left(X ; r ; L, d_{2} ; H\right)$. We will say that Condition (B) is satisfied for $M$ if $h^{0}(X, K \otimes L) \leq$ $d_{2}-2$.

Assume that Condition (B) is satisfied for the line bundle $M$; fix a general subset $Z \subset X$ with $\operatorname{card}(Z)=d_{2}$ and any subset $A$ of $Z$ with $\operatorname{card}(A)=d_{2}-2$. By the Cayley-Bacharach condition (see [C, Section 1], if $r=2$, [T] for the general case, and, exactly in the setting of our paper, [BB, Section 1] for $r=2$, [BB, Section 3] for arbitrary rank) the general extension (1) has as middle term $E$ a vector bundle. Since $X$ is integral, moving $Z$ we obtain as middle terms in the extensions an irreducible family (not uniquely parametrized) of bundles with rank $r$ and Chern classes $L$ and $d_{2}$; this is a consequence of the theory of the Relative Ext-Functors proved in [BPS]: the total space of all the extensions is a vector bundle with as base the space parametrizing all admissible $Z$ 's. Since stability is an open condition, we obtain a family of stable bundles (perhaps empty). We call its image in $M\left(X ; r ; c_{1}, c_{2} ; H\right)$ (after the identification of this moduli space -with $M\left(X ; r ; L, d_{2} ; H\right)$ obtained by tensoring with $M$ ) a good stratum for the line bundle $M$. Since the symmetric products of an integral quasi-projective variety are integral, every good stratum is integral, that is its closure in the Zariski topology is an integral scheme. Now we can state Condition (C).

Condition (C): There is a real line bundle $M$ such that $Y$ is contained in a good stratum for the line bundle $M$.

Since the complement of a codimension 1 submanifold in a connected differentiable manifold may be disconnected, the following axiom seems to be very important.

Condition (D) (for the real line bundle $M$ ): For a reduced finite set $Z \subset X$, let $\Pi(Z)$ be the set of all vector bundles fitting in (1) with the fixed $Z$. Let $S(Z)$ be the open subset of $\Pi(Z)$ formed by the stable bundles. A priori $S(Z)$ may be empty. Condition (D) is satisfied if for a dense set of all $Z \subset X$, with $Z$ reduced and $\operatorname{card}(Z)=d_{2}, \Pi(Z) \backslash S(Z)$ has codimension at least two in $\Pi(Z)$.

Now we discuss these conditions and introduce several technical conditions (Conditions (AB1), (AB2), (C0), (D0), (D1), (D2)) which may help to check in the examples conditions (A), (B), (C) and (D).

Condition (AB1) (for the line bundle $M$ ): Fix a real line bundle $M$ on $X$ and take $L, d_{2}$ given by (2). Assume $d_{2}-2 \geq \max \left\{h^{0}(X, K \otimes L), h^{0}(X, K-L), p_{8}\right\}$.

Condition (AB2) (for the line bundle $M$ ): Fix a real line bundle $M$ on $X$ and take $L, d_{2}$ given by (2). Assume $L$ ample, $h^{0}(L)>0$ and Condition (B) for the line 
bundle $M$.

Notice that by Kodaira vanishing Condition (AB2) implies Condition (AB1). We will see in Remark 1.2 that if either $r=2$ or $p_{g}=0$ the weaker forms of Conditions (B), (AB1) and (AB2) with $d_{2}$ instead of $d_{2}-2$ imply that our $Y$ will be smooth; the technical assumption with $d_{2}-2$ instead of $d_{2}$ will be used in an essential way in step (e) of the proof of Theorem 1.6, Theorem 1.7 and Theorem 1.8. Fix a good stratum $Y$ for the line bundle $M$ and $F \in Y$. Set $E:=F \otimes M$. By the definition of good stratum $E$ fits in an exact sequence (1) and hence we have $h^{0}(E) \geq r-1$. By Riemann-Roch we have $\chi(E)=r \chi+(L(L-K)) / 2-d_{2}$; by Serre duality $h^{2}(E)=h^{0}\left(K \otimes E^{*}\right)$. We dualize (1) and twist the corresponding exact sequence by $K$. We obtain the following exact sequence:

$$
0 \rightarrow K \otimes L^{*} \rightarrow K \otimes E^{*} \rightarrow(r-1) K \rightarrow K \otimes O_{z} \rightarrow 0
$$

To compute the cohomology groups of $E$ and the dimension of certain related families of bundles, one would like to know the rank of the linear map

$$
H^{0}(X,(r-1) K) \rightarrow H^{0}\left(Z, K \otimes O_{Z}\right)
$$

induced by the last map in (3). For general $Z$ one expects that this linear map is injective if $d_{2}<(r-1) p_{g}$ and surjective if $d_{2} \geq(r-1) p_{g}$. This is obvious if either $r=2$ or $p_{g}=0$. Hence we state the following numerical condition which implies Condition (C).

Condition (C0): There is a real line bundle $M$ such that, with the notation of (2), we have $d_{2} \geq(r-1) p_{g}, d_{2} \geq r \chi+2+(L(K+L)) / 2$, and such that $h^{0}\left(K \otimes L^{*}\right)=0$.

Condition $(\mathrm{C})$ is very restrictive; for instance by [ $\mathrm{Li}$, Theorem 0.1 ], it cannot be satisfied for large $c_{2}$ when $X$ is a surface of general type.

REMARK 1.2. Fix an integer $d_{2} \geq \max \left\{h^{0}(X, K \otimes L), h^{0}(X, K-L), p_{g}\right\}$ and a general $Z \subset X$ with $\operatorname{card}(Z)=d_{2}$. Assume either $r=2$ or $p_{8}=0$. Fix the line bundle $M$ and use the notation of (1) and (2). Assume $E$ locally free (for instance assume Condition (B) and that $E$ is the general sheaf give, by an extension (1)). Here we will check the smoothness of the formal deformation space of $[E]$ and hence, if $E$ is $H$-stable, that $E$ is a smooth point of $M\left(X ; r ; L, d_{2} ; H\right)$. Let $\operatorname{End}^{0}(E)$ be the sheaf of traceless endomorphisms of $E$. We have $\operatorname{End}(E) \cong \operatorname{End}^{0}(E) \oplus O$. By formal deformation theory it is sufficient to check that $h^{2}\left(\operatorname{End}^{0}(E)\right)=0$, that is that $h^{2}($ End $(E))=p_{g}$. We tensor (1) with $E^{*}$ and use that $Z$ has codimension 2 in $X$. We obtain:

$$
\begin{aligned}
p_{g} & \leq h^{2}(\operatorname{End}(E)) \leq(r-1) h^{2}\left(E^{*}\right)+h^{2}\left(E^{*} \otimes I_{Z}(L)\right) \\
& =(r-1) h^{2}\left(E^{*}\right)+h^{2}\left(E^{*} \otimes L\right)=(r-1) h^{0}(E \otimes K)+h^{0}(E \otimes(K-L))
\end{aligned}
$$




$$
\begin{aligned}
& \leq(r-1)^{2} p_{g}+(r-1) h^{0}\left(I_{Z}(K+L)\right)+(r-1) h^{0}(K-L)+h^{0}\left(I_{Z}(K)\right) \\
& =(r-1)^{2} p_{g} .
\end{aligned}
$$

Since either $r=2$ or $p_{g}=0$ by assumption, we conclude.

To check the very strong Condition (D) in the examples, we will give the following criteria for $r=2$ (see Condition (D0) and Lemma 1.3) and $r=3$ (see Conditions (D1) and (D2) and 1.5). For simplicity we will assume $q(X)=0$, since in the examples considered in the third section we have $q(X)=0$. Our feeling is that if $q(X)>0$ and $X$ has Kodaira dimension $\geq 0$ the real locus of 'most' moduli spaces is disconnected and the number of its connected components should not be bounded just in terms of the number of connected components of $X(\mathbb{R})$. However, we do not know how to construct large numbers of examples.

Condition (D0): Assume $q(X)=0, r=2$ and the existence of a real line bundle $M$ such that, with the notations of (2), we have $d_{2} \geq 2+h^{0}(L-R)$ for every $R \in \operatorname{Pic}(X)$ with $2(H R) \geq H L$.

Condition (D1): Assume $q(X)=0, r=3$ and the existence of a real line bundle $M$ such that, with the notations of (2), we have $d_{2} \geq 2+h^{0}(L-R)$ for every $R \in \operatorname{Pic}(X)$ with $3(H R) \geq H L$.

Condition (D2): Assume $q(X)=0, r=3$ and the existence of a real line bundle $M$ such that, with the notations of (2), we have $d_{2} \geq 2+2 h^{0}(L-A)$ for every $A \in \operatorname{Pic}(X)$ with $3(H A) \geq 2(H L)$.

LEMMA 1.3. Assume $r=2, h^{0}(K-L)=0$ and Condition (D0) for $Y$. Then $Y$ satisfies Condition (D).

PROOF. If $r=2$ a vector bundle $E$ fitting in (1) is $H$-stable if and only if we have $h^{0}\left(E \otimes R^{*}\right)=0$ for every $R \in \operatorname{Pic}(X)$ with $2(R H) \geq L H$. Fix $R \in \operatorname{Pic}(X)$ with $2(R H) \geq L H$ and tensor (1) by $R^{*}$. We obtain that $h^{0}\left(E \otimes R^{*}\right)=0$ if the restriction map $r: H^{0}(L-R) \rightarrow H^{0}(Z,(L-R) \mid Z)$ is injective. By the generality of $Z$ this is true (for the fixed $R$ ) if $d_{2} \geq h^{0}(L-R)$. We claim that if $d_{2} \geq 2+h^{0}(L-R)$ in the set of all extensions with locally free $E$ the set $\Gamma$ of non $H$-stable ones has codimension at least 2 . Fix a general $Z^{\prime} \subset X$ with $\operatorname{card}\left(Z^{\prime}\right)=\operatorname{card}(Z)-2$. Since $\operatorname{card}\left(Z^{\prime}\right) \geq h^{0}(L-R)$, the restriction map $t: H^{0}(L-R) \rightarrow H^{0}\left(Z^{\prime},(L-R) \mid Z^{\prime}\right)$ is injective. Hence for every finite scheme $Z$ containing the reduced scheme $Z^{\prime}$ the restriction map $H^{0}(L-R) \rightarrow H^{0}(Z,(L-R) \mid Z)$ is injective. The subset of the symmetric product $S^{d_{2}}(X)$ formed by all $Z$ with $\operatorname{card}(Z)=d_{2}, Z$ not containing any such $Z^{\prime}$ has dimension at most $2 d_{2}-2$. Notice that for all $A, B \subset X$ with $\operatorname{card}(A)=\operatorname{card}(B)=d_{2}$ and $h^{0}\left(K \otimes L \otimes I_{A}\right)=h^{0}\left(K \otimes L \otimes I_{B}\right)=0$ we have $\operatorname{dim}\left(\operatorname{Ext}^{1}\left(L \otimes I_{A}, O\right)=\operatorname{dim}\left(\operatorname{Ext}^{1}\left(L \otimes I_{B}, O\right)\right.\right.$. Hence we obtain the claim. The claim 
holds for the same reason for finitely many $R$ 's. Since $q(X)=0$ by condition (D0), the lemma follows.

1.4 Here we consider conditions (D1) and (D2). Hence we assume $r=3$ and $q(X)=0$. Applying the functor Hom to (2) (with $r=3$ ) we obtain the following exact sequence:

$$
0 \rightarrow L^{*} \rightarrow E^{*} \rightarrow 2 O_{X} \rightarrow O_{Z} \rightarrow 0
$$

Recall that to check the stability (in the sense of Mumford and Takemoto) of a rank 3 vector bundle $E$ it is sufficient to check the corresponding numerical condition for line bundles included in $E$ and for line bundles, $A$, with a non zero map $E \rightarrow A$, that is with $A^{*}$ subsheaf of $E^{*}$. Now we use (1) and the tensor power of (4) with $L$. Exactly as in the proof of Lemma 1.3 we see that Condition (D) is satisfied (with no unstable real bundle) if the Conditions (D1) and (D2) are satisfied.

REMARK 1.5. In the rank 2 case on $P^{2}$ and for all surfaces with $-K$ sufficiently positive (see for the case of $F_{e}$ the discussion of Condition (A) in the last part of 2.2) a bundle is $H$-stable if and only if it is simple. When this is the case, the computations made in Remark 1.2 allow one to bypass Condition (D0).

The main results are the following theorems: Theorem 1.6, Theorem 1.7 and Theorem 1.8.

THEOREM 1.6. Fix $X, c_{1}, c_{2}, H$ satisfying Conditions (A), (B), (C) and (D) with respect to a $\sigma$-invariant connected Zariski open subset $Y$ of $M\left(X ; 2 ; c_{1}, c_{2} ; H\right)_{\mathrm{reg}}$ and the line bundle $M$. Assume $X(\mathbb{R})=\emptyset$. Then $Y(\sigma)$ is connected.

THEOREM 1.7. Fix $X, c_{1}, c_{2}, H$ satisfying Conditions (A), (B), (C) and (D) with respect to a $\sigma$-invariant connected Zariski open subset $Y$ of $M\left(X ; 2 ; c_{1}, c_{2} ; H\right)_{\text {reg }}$ and the line bundle $M$. Assume $r \geq 3$. Assume either $X(\mathbb{R})=\emptyset$ or $X(\mathbb{R})$ connected. Then $Y(\sigma)$ has at most 2 connected components and each of these components intersects $M\left(X ; r ; c_{1}, c_{2} ; H\right)$ (real). If either $X(\mathbb{R})=\emptyset$ or $d_{2}:=c_{2}+M c_{1}+M^{2}$ is even and at least 4 , then $Y(\sigma)$ is connected.

THEOREM 1.8. Fix $X, c_{1}, c_{2}, H$ satisfying Conditions (A), (B), (C) and (D) with respect to a $\sigma$-invariant connected Zariski open subset $Y$ of $M\left(X ; 2 ; c_{1}, c_{2} ; H\right)_{\text {reg }}$ and the line bundle $M$. Assume $r \geq 3$. Assume that $X(\mathbb{R})$ has $s \geq 2$ connected components and that $d_{2}:=c_{2}+M c_{1}+M^{2} \geq 2 s+2$. Then there is a $\sigma$-invariant connected

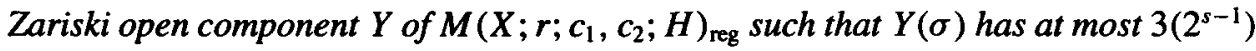
connected components, each of them intersecting $M\left(X ; r ; c_{1}, c_{2} ; H\right)_{\mathrm{reg}}(\mathrm{real})$. 
As a philosophical justification of the axioms, fix an integer $d \geq 2$ and let $R_{d}[t]$ be the set of degree $d$ real polynomials. Consider the set $T \subset R_{d}[t]$ of polynomials without multiple roots and the set $T_{1} \subset R_{d}[t]$ of polynomials with at most one double root (hence real, if any) and no root with multiplicity at least 3. $T$ is not connected but $T_{1}$ is connected because $R_{d}[t] \backslash T_{1}$ has codimension at least 2 in $R_{d}[t]$. Notice that a real double root can be both a limit of a family of two distinct real roots and of a family of pairs of complex conjugate roots. Axiom (B) (see steps (c) and (e) of the proof of Theorem 1.6, Theorem 1.7 and Theorem 1.8) allows us to use $T_{1}$ instead of $T$ as our building blocks (using the extension (1)) of vector bundles.

PROOF (of Theorem 1.6, Theorem 1.7 and Theorem 1.8).

(a) For every $\sigma$-invariant subset $W$ of $M\left(X ; r ; c_{1}, c_{2} ; H\right)(\mathbb{C})$, set $W($ real $):=W \cap$ $M\left(X ; r ; c_{1}, c_{2} ; H\right)($ real $)$. Notice that if $X(\mathbb{R})=\emptyset$, then in Condition $(C)$ and in all other axioms which use (2) we have $d_{2}$ even because in the exact sequence (1) $Z$ is $\sigma$-invariant and $Z$ is reduced by Bertini theorem (see for instance [K]). Hence, taking as $Z$ pairs of conjugate points if $X(\mathbb{R}) \neq \emptyset$, the definition of good stratum implies $Y($ real $) \neq \emptyset$. For the same reason $\operatorname{card}(Z(\mathbb{R}))-d_{2}$ is always even if $Z$ is reduced.

(b) Since $Y$ (real) $\neq \emptyset$ we have $Y(\sigma) \neq \emptyset$. Since $Y$ is smooth and irreducible, the set $Y(\sigma)$ is a differentiable manifold of realdimension $\operatorname{dim}_{\mathrm{C}}(Y)$ which intersects every Zariski open subset $Y^{\prime}$ of $Y$ (see for instance [Si, Chapter I, Section 1]). Hence it is sufficient to take $Y^{\prime}$ instead of $Y$ and prove the same assertions for $Y^{\prime}(\sigma)$. For the same reason if $Y^{\prime}$ is open and dense in a smooth real scheme $Y^{\prime \prime}$ and $Y^{\prime}(\sigma)$ is connected, then $Y^{\prime \prime}(\sigma)$ is connected. If $Y^{\prime \prime} \backslash Y^{\prime}$ has complex codimension at least 2 in $Y^{\prime \prime}$, then $Y^{\prime}(\sigma)$ is connected if and only if $Y^{\prime \prime}(\sigma)$ is connected. It is for these properties of smooth differentiable manifolds that we consider $Y(\sigma)$ instead of $Y($ real $)$ in the statements of Theorem 1.6, Theorem 1.7 and Theorem 1.8. To work with $\sigma$-invariant bundles, read again the discussion on stability and $\sigma$-invariants sets, $Z$, made before Section 1.1.

(c) Let $T$ be the set of all reduced subsets, $Z$, of $X(\mathbb{C})$ with card $(Z)=d_{2}$ and such that for every $Z^{\prime \prime} \subset Z$ with $\operatorname{card}\left(Z^{\prime \prime}\right)=d_{2}-2$, we have $h^{0}\left(X, K_{X} \otimes L \otimes I_{Z^{\prime \prime}}\right)=0$. Let $T_{2}$ be the set of all 0-dimensional subschemes $Z$ of $X$ with length $(Z)=d_{2}, Z$ disjoint union of a reduced set of $d_{2}-2$ points and a length 2 subscheme, and such that for every $Z^{\prime \prime} \subset Z_{\text {red }}$ with $\operatorname{card}\left(Z^{\prime \prime}\right)=d_{2}-2$, we have $h^{0}\left(X, K_{X} \otimes L \otimes I_{Z^{\prime \prime}}\right)=0$. Set $T_{1}:=T \cup T_{2} . T, T_{1}$ and $T_{2}$ are non-empty by assumption (B) (and of course $T$ and $T_{1}$ are irreducible of dimension $2 d_{2}$ ).

(d) Fix $Z \in T$ with $Z \sigma$-invariant. By the definition of $T$ and an easy part of the proof of the Cayley-Bacharach theorem (see [T]) the set of all the extensions (1) has dimension not depending on $Z$. Let $\Pi(Z)$ be the projectivization (over $\mathbb{C}$ ) of this complex vector space; $\Pi(Z)$ parametrizes (perhaps not uniquely up to isomorphism) the set of sheaves fitting in a non trivial extension like (1). By Cayley-Bacharach 
(see [C, Section 1], if $r=2$, [T] for the general case, and, exactly in the setting of our paper, [BB, Section 1] for $r=2$, [BB, Section 3] for arbitrary rank) the open subset $U(Z)$ of $\Pi(Z)$ parametrizing vector bundles has as complement the union of $d_{2}$ hyperplanes if $r=2$, while if $r>2$ it has lower dimensional complement and it is connected (as claimed in [B2, 5.1.(b) and step (1) of the proof of 5.2]). If $r=2$ each of the $d_{2}$ hyperplanes of $\Pi(Z) \backslash U(Z)$ corresponds to a point of $Z$ and each of the hyperplanes of $\Pi(Z)(\mathbb{R}) \backslash U(Z)(\mathbb{R})$ corresponds to a point of $Z \cap X(\mathbb{R})$. Hence if $r=2$ and $X(\mathbb{R})=\emptyset, U(Z)(\mathbb{R})$ is connected. It is crucial for our proof for $r=2$ to have the condition $X(\mathbb{R})=\emptyset$. Varying $Z$ among the $\sigma$-invariant 'good' subset we obtain a certain parameter space, $G$. By the theory of the relative Ext-functors ([BPS]) the set of all extensions (1) is parametrized by a vector bundle $\wp$ over $G$; here we use that $\operatorname{dim}(\Pi(Z))$ is constant for $Z \in G$. Hence $\wp$ and $G$ have the same number of connected components. The real part of the fiber $\pi^{-1}(Z)$ of the fibration $\pi: \wp \rightarrow G$ is just $\Pi(Z)(\mathbb{R})$ and the part of the fiber giving locally free sheaves is $U(Z)(\mathbb{R})$. Hence we obtain Theorem 1.6 and the case $X(\mathbb{R})=\emptyset$ of Theorem 1.7.

(e) Assume $r \geq 3$ and that $X(\mathbb{R})$ has s connected components, say $A_{1}, \ldots, A_{s}$, with $s>0$. Set $b(Z):=\operatorname{card}\left(Z_{\text {red }} \cap X(\mathbb{R})\right)$ and $a_{j}(Z):=\operatorname{card}\left(Z_{\text {red }} \cap A_{j}\right)$. Assume $a_{i}(Z) \geq 2$ for some integer $i$. Fix the $\left(d_{2}-b(Z)\right) / 2$ pairs of complex conjugate points of $Z$; fix all the points in $Z \cap\left(X(\mathbb{R}) \backslash A_{i}\right)$; fix $a_{i}(Z)-2$ points of $Z \cap A_{i}$ and move the other two, say $P^{\prime}$ and $P^{\prime \prime}$, in the components $A_{i}$ of $X(\mathbb{R})$ until they coincide, obtaining a double point, $z$, with support on $A_{i}$. Notice that by its definition $A_{i}$ is a connected 2-dimensional differentiable manifold. Set $Z^{\prime}:=Z \backslash\left\{P^{\prime}, P^{\prime \prime}\right\}$, $Z^{\prime \prime}:=Z^{\prime} \cup z$. Call $\left\{Z_{t}\right\}$ the family of deformations of $Z$ which converges to $Z_{0}:=Z^{\prime \prime}$ and call $\left\{P_{t}^{\prime}\right\}$ and $\left\{P_{t}^{\prime \prime}\right\}$ the corresponding deformations of $P^{\prime}$ and $P^{\prime \prime}$. By Conditions (B) and (C) we may apply the Cayley-Bacharach condition to $Z^{\prime \prime}$, that is $Z^{\prime \prime} \in T_{1}$, and to each $Z_{t}$. We may see $z$ as a limit of a pairs of complex conjugate points, say $\left\{Q_{t}^{\prime}, Q_{t}^{\prime \prime}\right\}$. Set $W_{t}:=Z^{\prime} \cup\left\{Q_{t}^{\prime}, Q_{t}^{\prime \prime}\right\}$. By Condition (C) each $W_{t}$ satisfies the CayleyBacharach condition. Since each $U\left(Z_{t}\right)(\mathbb{R}), U\left(W_{t}\right)(\mathbb{R})$ and $U\left(Z^{\prime \prime}\right)(\mathbb{R})$ is connected $(r \geq 3)$ we obtain that every connected component of $M\left(X ; r ; c_{1}, c_{2} ; H\right)(\sigma)$ contains a bundle fitting in an exact sequence (1) with some $Z$ with $0 \leq a_{j}(Z) \leq 1$ for every $j$. Furthermore, if we fix $s$ integers $t_{1}, \ldots, t_{s}$ with $0 \leq t_{i} \leq 1$ for every $i$ and $d_{2}-\sum_{1 \leq i \leq s} t_{i}$ even, there is at most one connected component of $M\left(X ; r ; c_{1}, c_{2} ; H\right)(\sigma)$ containing a bundle corresponding to a set $Z$ with $a_{i}(Z)=t_{i}$ for every $i$. Since $d_{2}-\sum_{1 \leq i \leq s} a_{i}(Z)$ is even for every $\sigma$-invariant $Z$, we obtain Theorem 1.8. For Theorem 1.7 notice that if either $X(\mathbb{R})=\emptyset$ or $s=1$ and $d_{2}$ is even, then we reduce to the case $t_{i}=0$ for every $i$.

We end the section with an easy application to our problem of the deep results proved in [GL] and [Li].

PROPOSITION 1.9. Fix a smooth complete algebraic surface $X$ defined over $\mathbb{R}$ and 
two real line bundles $c_{1}$ and $H$ on $X$ with $H$ ample. Let $M\left(X ; 2 ; c_{1}, c_{2} ; H\right)^{+}$be the compactification of $M\left(X ; 2 ; c_{1}, c_{2} ; H\right)$ parametrizing equivalence classes of $H$ semistable rank 2 torsion free sheaves on $X$ with Chern classes $c_{i}$. Then for every integer $k \geq 0$ there is an integer $d(k)$ such that if $c_{2} \geq d(k), M\left(X ; 2 ; c_{1}, c_{2} ; H\right)^{+}($real $)$ is locally $k$-connected around each of its points parametrizing a stable sheaf.

PROOF. Fix an integer $d$ (depending only on $X, H$ and $c_{1}$ ) for which [Li, Theorem 0.1 , holds, that is the following property holds for every $c_{2} \geq d$. Fix a stable sheaf $E \in M\left(X ; 2 ; c_{1}, c_{2} ; H\right)^{+}(\mathbb{R})$. By [Li, Theorem 0.1 ], and the choice of $d$, $M\left(X ; 2 ; c_{1}, c_{2} ; H\right)^{+}$is a locally complete intersection at $E$ with the expected dimension $\chi\left(\operatorname{End}^{0}(E)\right)=4 c_{2}-c_{1}^{2}+3\left(1-\chi\left(O_{X}\right)\right)$. Furthermore, by [Li, Lemma 1.3], at the point parametrizing $E$ the scheme $M\left(X ; 2 ; c_{1}, c_{2} ; H\right)^{+}$has Zariski tangent space with dimension at most $\chi\left(\operatorname{End}^{0}(E)\right)+a\left(X, H, c_{1}\right)$, where $a\left(X, H, c_{1}\right)$ is a constant depending only from $X, H$ and $c_{1}$, but independent from $c_{2}$ and the choice of $E$. Hence for large $d(k) \geq d$ the thesis follows from [H, Korollar 1.3] (or later generalizations) and the Smith exact sequence (see for instance [BR, Appendix C]).

\section{An example}

In this section we will give an example of a surface $X$ with very good moduli schemes (that is $M\left(X ; 2 ; c_{1}, c_{2} ; H\right)$ integral, everywhere smooth and with a Zariski open $\sigma$-invariant subscheme $Y$ satisfying Conditions (A), (B), (C) and (D)), but with $M\left(X ; 2 ; c_{1}, c_{2} ; H\right)(\sigma)$ not connected. We fix an elliptic curve $C$ defined over $\mathbb{R}$ and with $C(\mathbb{R})$ disconnected (for instance $C$ given by an equation $y^{2}=x^{3}+a x+b$ with $x^{3}+a x+b$ with 3 real roots). Set $X:=C \times P^{1}$ with $P^{1}$ with its usual structure, that is $P^{1}(\mathbb{R})$ a circle. Thus $s=2, X(\mathbb{R})=A_{1} \cup A_{2}$ with each $A_{i}$ real torus. Let $\pi_{1}: X \rightarrow C$ and $\pi_{2}: X \rightarrow P^{1}$ be the projections. We have $\operatorname{Pic}(X) \cong \pi_{1}^{*}(\operatorname{Pic}(C)) \oplus \pi_{2}^{*}\left(\operatorname{Pic}\left(P^{1}\right)\right) \cong \pi_{1}^{*}(\operatorname{Pic}(C)) \oplus Z$ and we will write every $R \in \operatorname{Pic}(X)$ as $R=(U, a)$ with $U \in \operatorname{Pic}(C)$ and $a \in Z$. The cohomology groups of $(U, a)$ are computed by Künneth's formula. In particular $h^{0}((U, a)) \neq 0$ only if $a \geq 0$. If $a \geq 0$ and $\operatorname{deg}(U)>0$ we have $h^{0}((U, a))=(a+1) \operatorname{deg}(U)$ and $h^{i}((U, a))=0$ for $i>0$. We have $K \cong\left(O_{C},-2\right)$ and $h^{0}(-K)=3$. We take as polarization $H$ a line bundle $(R, 1)$ with $\operatorname{deg}(R)=1$; however, there is no essential modification for different polarizations, that is if we take $H=(R, x)$ with $\operatorname{deg}(R)>0$ and $x>0$. Fix $L=(U, a) \in \operatorname{Pic}(X)$ with $u:=\operatorname{deg}(U) \geq 3$ and $a>0$. Hence $h^{0}(K+L)=(a-1) u=h^{0}(L)-2 u$. First we will check that every $M\left(X ; 2 ; c_{1}, c_{2} ; H\right)$ is everywhere smooth. Fix $F \in M\left(X ; 2 ; c_{1}, c_{2} ; H\right)$. Since $F$ is stable, it is simple, that is every homomorphism $F \rightarrow F$ is a homothety. Thus, since $-K$ is effective, $h^{0}(\operatorname{Hom}(F, F \otimes K))=0$. By Serre duality we have $h^{2}(\operatorname{End}(F))=0$. Hence by deformation theory (see for instance [Bru]) $F$ is a smooth point of $M\left(X ; 2 ; c_{1}, c_{2} ; H\right)$. 
Fix an integer $d_{2}$ with $(a-1) u+2 \leq d_{2} \leq(a+1) u$. Hence we have Condition (AB1). Consider a locally free rank 2 bundle fitting in the extension (1) with Chern classes given by (2) for $r=2$. Since $d_{2} \leq h^{0}(L)$, if $Z$ is general we have $\chi(E) \geq 1$, $h^{1}(E)=h^{2}(E)=0$ and $E$ is in a good stratum. Thus we see that there is a good stratum and that Condition (C) is satisfied. Now we will check Condition (D). Fix a bundle $E$ fitting in the extension (1) and assume that $E$ is not stable. Hence there is $W \in \operatorname{Pic}(X), W=(V, b)$ with a non zero homomorphism $t: W \rightarrow E$ and $2 \operatorname{deg}(V)+2 b \geq u+a$. Hence there is no non trivial map $W \rightarrow O$. Thus the homomorphism $t$ induces a non trivial map $t^{\prime}: W \rightarrow I_{Z}(L)$, that is $h^{0}\left(I_{Z}(L-W)\right) \neq$ 0 . Since $d_{2} \geq(a-1) u+2$ and $2 \operatorname{deg}(V)+2 b \geq u+a$, this is false for sufficiently general $Z$. Thus Condition (D) is satisfied. Notice that the complex dimension of the vector space of all the extensions of $I_{Z}(L)$ by $O$ does not depend on $Z$ and is the same even for unreduced $Z$. Thus we check easily using [BPS] the well-known fact that $M\left(X ; 2 ; L, d_{2} ; H\right)$ is integral; the same argument was used in the definition of good stratum and in step (d) of the proof of Theorem 1.6, Theorem 1.7 and Theorem 1.8. Let $M(0)$ (respectively $M(1)$ ) be the Zariski open subset of $M\left(X ; 2 ; L, d_{2} ; H\right)$ formed by the stable bundles fitting in (1) with $Z$ reduced (respectively card $\left(Z_{\text {red }}\right) \geq d_{2}-1$ ). We see easily that $M\left(X ; 2 ; L, d_{2} ; H\right) \backslash M(1)$ has codimension at least 2 . Hence, as in Step (b) of the proof of Theorem 1.6, Theorem 1.7 and Theorem 1.8, by the smoothness of $M\left(X ; 2 ; L, d_{2} ; H\right)$ it is sufficient to show that $M(1)(\mathbb{R})$ is not connected, that is we may ignore bundles corresponding to a $\sigma$-invariant 0 -cycle, $Z^{\prime \prime}$, with card $\left(Z_{\text {red }}^{\prime \prime}\right)<d_{2}-1$. Fix $E^{\prime}$ and $E^{\prime \prime}$ in $M(0)(\mathbb{R})$, say given bȳ reduced subschemes $Z^{\prime}$ and $Z^{\prime \prime}$ and extensions (1), with $\operatorname{card}\left(Z^{\prime} \cap A_{1}\right)-\operatorname{card}\left(Z^{\prime \prime} \cap A_{1}\right)$ odd. We claim that $E^{\prime}$ and $E^{\prime \prime}$ are in different connected components of $M(1)(\mathbb{R})$. The bundles $E^{\prime}$ and $E^{\prime \prime}$ are in different components of $M(0)(\mathbb{R})$ by the constancy of the dimension of the Ext ${ }^{1}$-group and the fact that $\operatorname{card}\left(A_{1} \cap Z_{t}\right)$ is preserved for a continuous family of bundles $E_{t}$ in $M(0)(\mathbb{R})$ with $E_{t}$ given by $Z_{t}$. Fix $F \in(M(1)(\mathbb{R}) \backslash M(0)(\mathbb{R}))$ with $F$ limit of a continuous family $\left\{E_{t}\right\}$ as above and $F$ given by $Z^{*} \cup z$ with length $(z)=2$ and $\operatorname{card}\left(Z^{*}\right)=d_{2}-2$. Since $\operatorname{card}\left(Z_{t} \cap A_{1}\right)-\operatorname{card}\left(Z^{*} \cap A_{1}\right)$ is even, we see that $E^{\prime}$ and $E^{\prime \prime}$ are in different components of $M(1)(\mathbb{R})$, as wanted.

\section{Segre-Hirzebruch surfaces}

In this section we show how to check the axioms in the case of the Segre-Hirzebruch surfaces, $F_{e}, e \geq 0$.

3.1. Here we consider the case of the Segre-Hirzebruch surfaces, $F_{e}, e \geq 0$, with the usual real structure, that is with the unique real structure with $F_{e}(\mathbb{R}) \neq \emptyset$; if e-is even $F_{e}(\mathbb{R})$ is a torus; if $e$ is odd $F_{e}(\mathbb{R})$ is a Klein bottle ([Si, Chapter VI, Proposition 1.2]). For the other real structures, see Section 3.2. Set $\Pi:=P^{1}$ and 
let $\pi: X \rightarrow P^{1}$ be the ruling (unique, except for the quadric surface $F_{0}$ ). For the results used here on $\operatorname{Pic}\left(F_{e}\right)$, see [Ha, Chapter V, Section 2], and [GH, pp. 523-525]. Since $\operatorname{Pic}\left(F_{e}\right) \cong \mathbb{Z}^{2}$, the numerology for all possible $r, e, H, c_{1}$ is so complicated that not even the existence of rank 2 stable bundles for a given arbitrary quadruple ( $e$, $H, c_{1}, c_{2}$ ) is completely known (see [Q] and the references in [B1] for many cases). Hence we will restrict (except for Conditions (B), (D1) and (D2)) to the case of rank 2 bundles, we will make strong numerical assumptions which will simplify the life and we will give only a partial picture of the existence of stable bundles and the irreducibility of $M\left(F_{e} ; 2 ; c_{1}, c_{2} ; H\right)$. The general picture should be very similar. We take as base defined over $\mathbb{R}$ of $\operatorname{Pic}\left(F_{e}\right)$ a section $h$ of the ruling with minimal selfintersection and a fiber $f$ of the ruling. Hence $h^{2}=-e, h f=1$ and $f^{2}=0$. We have $K=-2 h-(e+2) f$. The bundle $x h+y f$ is ample if and only if $x>0$ and $y>x e$. Since $\pi_{*}(O(u h+v f)) \cong \bigoplus_{0 \leq j \leq u}\left(O_{\Pi}(v-j e)\right)$ for every $u \geq 0$, by the Leray spectral sequence of $\pi$ we have $H^{1}(O(u h+v f))=0$ if $u \geq 0$ and $v \geq e x-1$. Set $L:=a h+b f, H:=c h+d f, H$ ample. Assume $L$ ample, that is $a>0, b>e f$; hence we have $h^{1}(L)=0$. We have:

$$
\begin{aligned}
h^{0}(K+L) & =1+(L 2) / 2=1+a(2 b-e a) / 2 \\
h^{0}(L) & =h^{0}(K+L)-(K L)=h^{0}(K+L)+2(b-a e)+a(e+2)
\end{aligned}
$$

Hence we see exactly when condition (B) is satisfied. If $M=\alpha h+\beta f$ we have

$$
d_{2}=c_{2}+(r-1)(\alpha \beta+\alpha b-e \alpha a)+r(r-1)\left(2 \alpha \beta-e \alpha^{2}\right) / 2 .
$$

Thus condition (B) is satisfied for $M$ if and only if $c_{2} \geq 3+a(2 b-e a) / 2-(r-$ 1) $(\alpha \beta+\alpha b-e \alpha a)-r(r-1)\left(2 \alpha \beta-e \alpha^{2}\right) / 2$. Here $c_{1}:=L-r M$. The condition that the corresponding bundle $E$ has $h^{1}(E)=0$ is $d_{2} \leq h^{0}(L)$, or equivalently, by condition (B)

$$
h^{0}(K+L)+2 \leq d_{2} \leq h^{0}(K+L)+2(b-a e)+a(e+2) .
$$

For conditions (D0) and (D1) we fix $R:=x h+y f$; hence $R H=-e x c+y c+x d$. Hence $r(R H) \geq(L H)$ if and only if

$$
-r x c+r d x-r e y c \geq c b+d a-e b c .
$$

We have $h^{0}(L-R) \neq \emptyset$ if and only if $x \leq a$ and $b-x \geq e(a-x)$. The same computations apply for condition (D2). Notice that by deformation theory and Serre duality (see for instance [B1]) the moduli space is smooth at the points with $h^{0}(\operatorname{Hom}(E, E \otimes K))=0$. If $E$ is $H$-stable for some polarization $H$ we have $h^{0}(\operatorname{Hom}(E, E \otimes K))=0$ if $-K$ is effective or ample, that is if $0 \leq e \leq 2$. For every 
$e \geq 0$ and every $H$-stable bundle $E$ we have $h^{0}(\operatorname{Hom}(E, E \otimes K))=0$ for a good choice of a polarization $H$, that is if we have $K H<0$, that is

$$
(2 h+(e+2) f)(c h+d f)>0, \text { that is } 2 d-c e+2 e>0 .
$$

In a few very particular cases (for instance for the quadric $F_{0}$ with $H=-K$ ) the arithmetic simplifies drastically and everything was previously known. In summary, for $r=2$ and fixed $e, H$ and $c_{1}$ everything (that is the existence and the dimension of a good stratum and cohomological properties of the general members of any good stratum) may be computed just using straightforward numerical calculations. If we do not fix $e, H$, and $c_{1}$ there is a 'large sector' of triples $\left(e, H, c_{1}\right)$ in which these computations may be done simultaneously in a uniform way. It seems to us that there is no statement (or at least no statement shorter than 10 pages) covering all cases.

3.2. $P^{2}$ has a unique real structure ([Si, Chapter VI, Proposition 1.1]). If the integer $e$ is odd the surface $F_{e}$ has a unique real structure ([Si, Chapter VI, Proposition 1.2]). If $e$ is even $F_{e}$ has a unique real structure with $F_{e}(\mathbb{R}) \neq \emptyset$ ([Si, Chapter VI, Proposition 1.2]). Here we assume $e$ even and take a real structure with $F_{e}(\mathbb{R})=\emptyset$. As shown in Section 3.1 by the cohomological discussion for the usual real structure, the real structure may enter in the picture only to say if $h$ or $f$ or both are real (we will see that $2 h$ and $2 f$ are always real), and, if real, if they have the usual structure of $P^{1}$ or no real point. If $e>0, h$ is unique and hence $\sigma$-invariant for any real structure. Thus $\pi$ is real. Since $K$ is always real, if $e>0$ and even, $2 f$ is real; here everything works as in Section 3.1 taking $H:=c h+d f$ with $d$ even. If $e=0$ it is sufficient to assume both $c$ and $d$ even.

\section{Acknowledgements}

The author was partially supported by MURST and GNSAGA of CNR (Italy). We would like to thank the referee for several useful suggestions.

\section{References}

[B1] E. Ballico, 'On moduli of vector bundles on rational surfaces', Arch. Math. 49 (1987), 267-272.

[B2] - 'Real moduli of complex objects: surfaces and bundles', Monatsh. Math. 115 (1993), 13-26.

[BB] E. Ballico and R. Brussee, 'On the unbalance of vector bundles on a blown-up surface', Technical Report, 1990.

[BPS] C. Banica, M. Putinar and G. Schumacher, 'Variation der globalen Ext in Deformationen komplexer Räume', Math. Ann. 250 (1980), 135-155. 
[BR] R. Benedetti and J.-J. Risler, Real algebraic and semi-algebraic sets (Hermann, Paris, 1990).

[Bru] J. Brun, 'Les fibrés de rank deux sur $P^{2}$ et leurs sections', Bull. Soc. Math. France 107 (1979), 457-473.

[C] F. Catanese, 'Footnotes to a theorem of I. Reider', in: Algebraic geometry, Proceedings, L'Aquila, 1988, Lecture Notes in Math. 1417 (Springer, Berlin, 1990) pp. 67-74.

[GL] D. Gieseker and J. Li, 'Irreducibility of moduli of rank two vector bundles on algebraic surfaces', J. Diff. Geometry 40 (1994), 23-104.

[GH] P. Griffiths and J. Harris, Principles of algebraic geometry (Wiley, New York, 1978).

[H] H. Hamm, 'Lokale topologische Eigenschaft komplexer Räume', Math. Ann. 191 (1971), 235-252.

[Ha] R. Hartshorne, Algebraic geometry (Springer, Berlin, 1977).

[K] S. Kleiman, 'The transversality of a general translate', Compositio Math. 28 (1974), 287-297.

[L] S. Langton, 'Valuative criteria for families of vector bundles on algebraic varieties', Ann. of Math. 101 (1975), 88-110.

[Li] J. Li, 'Kodaira dimension of moduli space of vector bundles on surfaces', Invent. Math. 115 (1993), $1-40$.

[Q] Z. Qin, 'Moduli spaces of stable rank-2 bundles on ruled surfaces', Invent. Math. 110 (1992), 615-625.

[Si] R. Silhol, Real algebraic surfaces, Lecture Notes in Math. 1137 (Springer, Berlin, 1985).

[T] A. N. Tyurin, 'Cycles, curves and vector bundles on an algebraic surface', Duke Math. J. 54 (1987), 1-26.

Department of Mathematics

University of Trento

38050 Povo (TN)

Italy

e-mail: ballico@science.unitn.it 